\title{
Seeing the Portal in Membrane-containing Bacteriophage PRD1 by Cryo-EM
}

\author{
Chuan Hong ${ }^{1,2}$, Xiangan Liu², Hanna M. Oksanen ${ }^{3}$, Joanita Jakana², Dennis H. Bamford ${ }^{3}$, Wah Chiu ${ }^{1,2}$ \\ 1. Graduate Program in Structural and Computational Biology and Molecular Biophysics, and \\ 2. Department of Biochemistry and Molecular Biology, Baylor College of Medicine, Houston, TX 77030 \\ USA \\ 3. Institute of Biotechnology and Department of Biosciences, University of Helsinki, FIN-00014 \\ Helsinki, Finland
}

The functional and structural knowledge of assembly principles of viruses, often dsDNA viruses composed of only proteins and nucleic acids, have extended our understanding of viral capsid maturation and genome packaging processes. Viruses with lipids possess an additional complexity when exploring the mechanistic and structural properties of such fundamental functions.

Bacteriophage PRD1 (Tectiviridae family) is the first membrane containing virus structure that has been solved by X-ray crystallography [1]. During viral replication, $\sim 30$ phage-specific proteins and host-derived lipids are assembled into a procapsid, a precursor of the mature virion. Of these proteins, as many as four, including ATPase P9, packaging efficiency factor P6, and integral membrane proteins P20 and P22, are needed to form the packaging complex, a unique feature located at one of the twelve vertices [2]. The packaging complex is essential to the virus life cycle as it is the structure that permits encapsidation of the $\sim 15 \mathrm{~kb}$ dsDNA genome during maturation. To date, the unique vertex still remains structurally elusive mainly due to technical challenges in identifying non-icosahedral features in a highly symmetrical virus particle.

Recent developments in imaging technology and novel algorithms developed for electron cryo-microscopy have provided more power in resolving non-icosahedrally organized features in icosahedral viruses, such as the genome packaging and release machinery of tailed bacteriophages $[3,4]$ and the portal in herpes simplex type-1 B capsid [5].

Here, we describe an advanced Cryo-EM image-processing algorithm (Fig. 1) that enabled the first-time revelation of the genome packaging complex structure with a membrane conduit at a unique vertex in tailless icosahedral membrane-containing bacteriophage PRD1. As a first step, icosahedral orientation was determined for each raw virus particle and icosahedral reconstruction was performed. Under very low threshold, a faint feature at the vertex was detectable and used as a starting point to determining the asymmetric orientation. The corresponding 3D mask of the faint feature was used to generate the library of 2D dynamic masks that sufficiently cover all possible orientations of the unique vertex and were applied to each raw virus particle. Using cross common line search in Fourier space, the generated library of each masked virus particle would be compared to the library of references generated from the initial faint feature. Since the unique vertex locates at one of the 12 vertices and has 5 possible attachments of the symmetry mismatch to the 5-fold, the search will pick the best orientation out of 60 possible choices (12 vertex locations * 5 attachments) based on the lowest phase residue. The map would be reconstructed without imposing icosahedral symmetry from the particles each with the best possible orientation. The refinement cycle was carried out iteratively, further improving the features of the unique vertex and generating the final converged map. 
We successfully applied this advanced algorithm to the reconstructions of not only bacteriophage PRD1 virion, but also its procapsid and packaging mutant particles. Without icosahedral symmetry imposition, the PRD1 virion structure at $12 \AA$ resolution showed that a packaging complex replaces the regular fivefold structure at a special vertex across membrane. The combined knowledge from the virion, the procapsid and the mutant structures revealed that the packaging ATPase P9 and the packaging efficiency factor P6 form a dodecameric portal complex external to the membrane compartment. The structure of the procapsid identified the hexameric arrangement of P20 and P22 embedded in the viral membrane, functioning as a membrane conduit for the DNA packaging and a nucleating site for the complex assembly. These structures allowed us to propose a pathway for the assembly and packaging process in membrane-containing bacteriophage PRD1.

\section{References:}

[1] Abrescia, NG, et al., Nature 432 (2004), 68-74.

[2] Gowen, B, et al., J Virol 77 (2003), 7863-7871.

[3] Jiang, W, et al., Nature 439 (2006), 612-616.

[4] Liu, X, et al., Nat Struct Mol Biol 17 (2010), 830-836.

[5] Rochat, RH, et al., J Virol 85 (2011), 1871-1874.

[6] Supported by grants from NIH (P41GM103832 and R01AI0175208), Robert Welch Foundation (Q1242), and Academy of Finland (271413, 255342 and 256518).

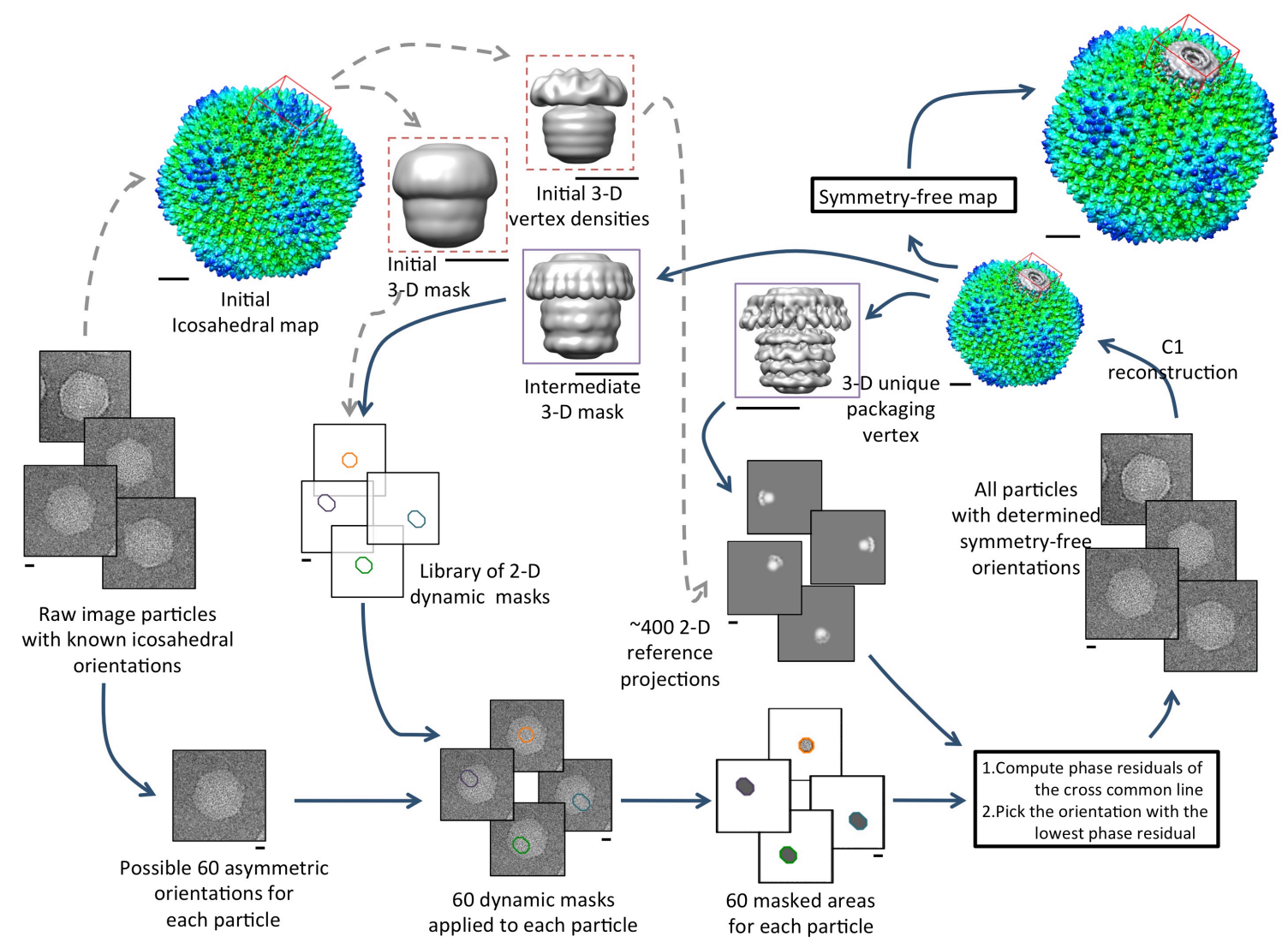

Figure 1. An illustration of the algorithm in reconstructing virus structures without icosahedral symmetry imposition. Scale bar $100 \AA$. 